\title{
Effect of Tourniquet use on Blood Loss, Pain, Functional Recovery, and Complications in Robot- assisted Total Knee Arthroplasty: A Prospective, Double-blinded, Randomized Controlled Trial
}

\section{Yahao Lai}

Sichuan University West China Hospital

\section{Hong Xu}

Sichuan University West China Hospital

\section{Qiang Su}

Sichuan University West China Hospital

\section{Xufeng Wan}

Sichuan University West China Hospital

\section{Mingcheng Yuan}

Sichuan University West China Hospital

Zongke Zhou ( $\square$ zhouzongke@scu.edu.cn )

Sichuan University West China Hospital https://orcid.org/0000-0002-9037-4756

\section{Research article}

Keywords: Robot-assisted total knee arthroplasty, tourniquet, blood loss, pain, functional recovery

Posted Date: November 15th, 2021

DOI: https://doi.org/10.21203/rs.3.rs-1064017/v1

License: (c) (1) This work is licensed under a Creative Commons Attribution 4.0 International License. Read Full License

Version of Record: A version of this preprint was published at Journal of Orthopaedic Surgery and Research on February 21st, 2022. See the published version at https://doi.org/10.1186/s13018-02202992-y. 


\section{Abstract}

Background: Robot-assisted total knee arthroplasty (TKA) has been largely studied to confirm its advantages in terms of accurate component positioning, microembolus formation, less blood loss and so on, but is currently usually performed under tourniquet due to its longer operative time than conventional TKA. The aim of this study was to estimate the effects of tourniquet use in robot-assisted TKA on blood loss, pain, functional recovery, and complications.

Methods: Patients scheduled for robot-assisted TKA were prospectively randomized into a tourniquet or non-tourniquet group (each $n=14$ ). The primary outcome measure was blood loss. The secondary outcome measures were operation time; visual analog scale (VAS) pain scores; time to achieve the first straight-leg raise; swelling of the thigh, knee, and calf; range of motion; Hospital for Special Surgery score; length of stay; and postoperative complications.

Results: There was no significant difference in total blood loss between the tourniquet and non-tourniquet groups (738.57 \pm 276.158 vs. $866.85 \pm 243.422 \mathrm{ml}, \mathrm{P}=0.061)$. The tourniquet group showed significantly lower intraoperative blood loss $(P<0.001)$, but higher hidden blood loss $(P=0.002)$. The non-tourniquet group showed better knee range of motion on POD 1-3 (all $P<0.001$ ), less thigh swelling on PODs 2 and $3(P<0.05)$, earlier straight-leg raising $(P=0.044)$, and shorter length of stay $(P=0.044)$. Thigh pain VAS score at 1 month after surgery was significantly greater in the tourniquet group $(P<0.001)$, as was knee pain during activity and at rest on PODs $2-3($ all $P<0.05$ ). The tourniquet group also showed a significantly higher rate of tension blisters $(28.8 \%$ vs. $7.1 \%, \mathrm{P}=0.038)$.

Conclusions: Tourniquet use during robot-assisted TKA dose not reduce total blood loss and it appears to increase postoperative pain, aggravate muscle injury, and prolong postoperative recovery.

Trial registration: ChiCTR, ChiCTR2100041800. Registered 5 January 2021, http://www.chictr.org.cn/index.aspx

\section{Introduction}

An estimated 30.8 million adults in the United States alone and 300 million individuals worldwide are living with osteoarthritis ${ }^{[1]}$. Total knee arthroplasty (TKA) has become the gold-standard treatment for patients with severe knee osteoarthritis whose pain was not controlled by other interventions ${ }^{[2]}$. The patients who need surgery with effective outcomes highlights the need for measures that increase the durability and longevity of prostheses.

Judicious choice of prosthetic components and careful positioning of the implant can prolong prosthesis life and improve patient satisfaction ${ }^{[3-6]}$. Robot-assisted TKA can allow more accurate component positioning ${ }^{[7,8]}$, particularly in difficult situations, such as when patients are obese or have suffered posttraumatic deformity or when patients are undergoing revision knee arthroplasty ${ }^{[9,10]}$. In addition, robotassisted TKA can reduce blood loss by avoiding the need to insert instrumentation into the intramedullary 
canal $^{[11]}$, which also reduces risk of operator error ${ }^{[12]}$ and microembolus formation ${ }^{[13]}$. Robot-assisted TKA may eliminate the requirement for a bloodless visual field that applies to the conventional procedure $^{[14]}$. The computer system can automatically select the osteotomy surface.

Nevertheless, robot-assisted TKA is associated with longer operation time ${ }^{[15]}$, especially before the clinician has gained experience, since the procedure involves a long learning curve $\mathrm{e}^{[16]}$. This longer operation time can lead to higher intraoperative blood loss, so robot-assisted TKA is typically performed with a tourniquet ${ }^{[17]}$.

This view seems to be contrary to that of studies on non robotic TKA. Most studies about tourniquet use in conventional TKA believe that tourniquet use can not reduce the total blood loss, and will lead to delayed recovery ${ }^{[5,17-21]}$. However whether it provides net benefits is controversial ${ }^{[21]}$, especially with advances in surgical techniques and perioperative management. In addition, the study of tourniquet use in robot-assisted TKA has not been reported. Therefore, we conducted a prospective trial in which patients receiving robot-assisted TKA were randomized into tourniquet and non-tourniquet groups, and we examined the influence of the tourniquet on blood loss, pain, functional recovery, and complications.

\section{Methods}

\section{Patients}

This study obtained the approval of the Ethics Committee and Institutional Review Board of our hospital, and informed patient consent for each enrolled patient was also obtained. The study was designed as a prospective, double-blinded, randomized controlled trial. Thirty eligible patients were enrolled in this study. Two of them were excluded because they did not meet the inclusion criteria.

Inclusion criteria were as follows: (i) age between 18 and 80 years, (ii) diagnosis of severe knee osteoarthritis that failed to be treated conservatively, (iii) investigator-assessed requirement for primary unilateral TKA, and (iv) full patient understanding of the benefits and risks of this study and written informed consent. Patients were excluded if they had (i) coagulation disorders, based on preoperative routine monitoring of Activated partial thromboplastin time (APTT), Prothrombin time (PT), Fibrinogen (FIB), Thrombin time (TT), (ii) body mass index $>40 \mathrm{~kg} / \mathrm{m}^{2}$, or (iii) history of lower limb fracture.

Patients were randomized into a tourniquet group and non-tourniquet group according to a computerized random sequence generator. The researcher collecting data was uninvolved in study procedures and was blinded to group allocation.

\section{Perioperative management}

The same surgeon performed all the operations, and patients received combined intravenous and inhalation anesthesia with a standardized regimen. A pneumatic tourniquet was applied to the tourniquet group at a pressure of $240 \mathrm{mmHg}$ thoughout the surgery. We relied on preoperative computed 
tomography (CT) of the hip, knee, and ankle to help the clinician decide the optimal prosthetic orientation in the robot-assisted system (YUANHUA-TKA). Procedures were performed with patients in the supine position, with the tibia and foot in a mobile boot. Femoral and tibial registration pins were used to register the leg position in the system at the start of the operation. We utilized a midline skin incision with a medial parapatellar approach for every patient. Bone cuts were made with robotic arm assistance based on the preoperative system plan. The surgeon could modify bone resection by using a dynamic tracking system to track alignment, flexion, extension gaps, and range of movement.

All patients received the same perioperative treatment strategies: tranexamic acid (TXA), pain management, thrombosis prevention, and functional rehabilitation. Celecoxib (200 mg) was administered twice a day from admission to 14 days after discharge. All patients received $2 \mathrm{~g}$ of intravenous TXA at 30 min before the incision and $1 \mathrm{~g}$ of intravenous TXA at 3 and 6 hours after the surgery. All patients received $5 \mathrm{mg}$ dexamethasone intravenously before TXA. For thrombosis prophylaxis, we administered $0.2 \mathrm{ml}$ of low-molecular-weight heparin (enoxaparin) at 8 hours after the procedure, which was increased to $0.4 \mathrm{ml}$ per day from the next day until discharge. Subsequently, apixaban was continued for two weeks.

Patients were discharged when (i) pain control was judged to be adequate, defined as a visual analogue scale (VAS) score < 3 , (ii) the patient could actively flex the knee to at least $90^{\circ}$, and (iii) the patient could walk independently with a walking aid.

\section{Primary outcomes}

The primary outcome was blood loss, defined to include total blood loss, intraoperative blood loss, drainage volume, and hidden blood loss. The total blood loss volume of each patient was calculated according to Nadler's formula ${ }^{[22]}$. Gross's formula was then used to calculate total blood loss based on total blood volume and hematocrit drop ${ }^{[23]}$. Intraoperative blood loss was estimated from the increase in gauze weight and the volume (excluding saline) in the negative pressure aspirator bottle. The drainage tube was removed at 24 hours after the operation, and the drainage volume was recorded. Postoperative blood loss was calculated from the drainage volume and dressing weight. Hidden blood loss was defined as total blood loss minus intraoperative and postoperative blood loss.

\section{Secondary outcomes}

Secondary outcomes were operation time; VAS scores; time to achieve the first straight-leg raise; swelling of thigh, knee and calf; range of motion; Hospital for special surgery (HSS); length of stay; and postoperative complications. These outcomes were assessed at admission, every morning on postoperative days (PODs) 1-3, on the day of discharge, and at one and three months postoperatively. On the mornings of PODs 1-3, VAS scores were used to assess thigh pain, knee pain at rest, and knee pain during activity (when knees were flexed to the maximum extent).

Postoperative complications were recorded and included tension blisters, ecchymosis, superficial infection, numbness of lower limbs, and deep vein thrombosis (DVT). DVT was monitored through a 
bilateral lower extremity deep venous color Doppler ultrasound examination on POD 1 and at 1 month postoperatively.

After returning to the ward from the post-anesthesia care unit, the patient was told to make his or her best effort to perform straight-leg raising, and the time needed for this was recorded by a nurse. Swelling in the lower limbs was assessed by measuring circumferences of the thigh (10 cm above the patella midline), knee (patella midline), and calf (10 cm below the patella midline).

All patients received physical therapy from the same rehabilitation physician on POD 1. On PODs 1-3, range of motion was measured using a goniometer based on how far the patient was able to flex the knee.

\section{Statistical analysis}

The minimal sample size was estimated based on a previously reported mean blood loss of $613 \mathrm{ml}$ for robot-assisted TKA ${ }^{[11]}$ and our definition of $200 \mathrm{ml}$ as the smallest clinically meaningful reduction in blood loss due to tourniquet use. These considerations, combined with a medium effect size of 0.5 , power of 0.8 , and alpha error of 0.2 , meant that at least 28 patients had to be enrolled in the study.

Data were reported as mean \pm standard deviation (SD) if they were normally distributed according to the Shapiro-Wilk test. Otherwise, data were reported as the median (25th percentile, 75th percentile). Intergroup differences in continuous variables were assessed for significance using Student's t-test or the Mann-Whitney test, depending on the type of data and whether they were normally distributed. Differences in categorical data were assessed using Fisher's exact test. Differences associated with $\mathrm{P}<$ 0.05 were considered significant. All analyses were performed in SPSS 26.0 (IBM, Armonk, NY, USA).

\section{Results}

\section{Participants}

Of the total 30 potentially eligible patients treated during the recruitment period, 2 were excluded because they did not meet the inclusion criteria. 28 patients were randomly and equally divided into two groups. There were no significant differences in clinico-demographic characteristics between the two groups (Table 1). No patients were lost to follow up (Fig. 1).

\section{Blood loss}

Between the tourniquet and non-tourniquet groups there was no significant difference in total blood loss (738.57 \pm 276.158 vs. $866.85 \pm 243.422 \mathrm{ml}, \mathrm{P}=0.061)$ or drainage volume $[145(100,180)$ vs. $95(23.75$, 210) $\mathrm{ml}, \mathrm{P}=0.071]$. The tourniquet group showed significantly lower intraoperative blood loss (134.17 \pm 57.191 vs. $308.67 \pm 63.122 \mathrm{ml}, \mathrm{P}<0.001)$, but significantly higher hidden blood loss (511.15 \pm 183.322 
vs. $366.00 \pm 171.281 \mathrm{ml}, \mathrm{P}=0.002$ ). No patient required blood transfusion at any time before discharge (Table 2).

\section{Secondary outcomes}

Although the non-tourniquet group underwent surgery for a significantly longer time $(117.57 \pm 33.184$ vs. $96.77 \pm 21.379 \mathrm{~min}, \mathrm{P}=0.005$ ), they achieved better knee range of motion on PODs 1-3 (Fig. 2A).

However, the two groups did not differ significantly in knee range of motion at later time points or in knee functional recovery based on HSS score.

On PODs 2-3, thigh swelling was more obvious in the tourniquet group than in the non-tourniquet group (Fig. 2B). At one month after surgery, thigh pain VAS scores were significantly higher in the non-tourniquet group (Fig. 3A). On PODs 2 and 3, but not at later time points, the tourniquet group reported more severe knee pain than the non-tourniquet group during activity and at rest (Fig. 3B-C).

A significantly higher number of patients in the tourniquet group $(n=4)$ experienced tension blisters than in the non-tourniquet group $(n=1)(P=0.038)$. All blisters resolved with medical treatment. The two groups did not differ significantly in rates of ecchymosis, numbness of lower limbs, superficial infection, or DVT (Table 3).

The non-tourniquet group took significantly less time to achieve the first straight-leg raise, and their length of stay was shorter [6 (6-7) days, $\mathrm{P}=0.044]$.

\section{Discussion}

In what appears to be the first study to explore whether the tourniquet is beneficial to patients undergoing robot-assisted TKA, our data suggest that using a tourniquet in robot-assisted TKA does not decrease total blood loss and instead leads to more severe pain, slower functional recovery, longer hospital stay, and more frequent complications.

Perhaps the most important finding of our study is that the tourniquet in robot-assisted TKA does not significantly decrease total blood loss. While the tourniquet appears to reduce intraoperative blood loss and shorten the operation, it fails to decrease hidden blood loss. Similarly, a study involving conventional TKA ${ }^{[24]}$ found that the tourniquet can effectively control intraoperative blood loss but not reduce postoperative or total blood loss. On the contrary, a randomized controlled trial suggested that tourniquet use during conventional TKA significantly decreases blood loss without adversely affecting early postoperative outcomes ${ }^{[25]}$. We may need a high-quality meta-analysis to assess whether the tourniquet can significantly reduce total blood loss.

Whatever the case, it seems clear enough that using a tourniquet during robot-assisted TKA increases hidden blood loss. Hidden blood loss arising from postoperative hyperfibrinolysis accumulates in the 
third anatomic space and has been associated with postoperative inflammation, lower limb swelling, subcutaneous ecchymosis, and pain ${ }^{[26]}$. Our swelling data are consistent with this. Since swelling is associated with quadricep weakness and slower gait ${ }^{[27]}$, interventions to reduce knee swelling after TKA may strengthen quadriceps and improve gait speed.

Numerous studies have shown that using a tourniquet during conventional TKA does not reduce total blood loss, but instead it appears to increase risk of more severe pain, longer length of stay, slower functional recovery, and complications ${ }^{[21,25,28-30]}$, similar to our results. Since robot-assisted TKA takes significantly longer than conventional surgery ${ }^{[31]}$, and since risk of tourniquet-associated complications increase with tourniquet time ${ }^{[32]}$, we suspect that at least some of the adverse effects of tourniquet use in robot-assisted TKA relate to the relatively long operation time.

Our study demonstrated that tourniquets are associated with more severe pain soon after robot-assisted TKA, especially pain in the thigh. In fact, the pain appears to be higher than that reported for tourniquet use during conventional TKA ${ }^{[33]}$. Pain in the early postoperative period can strongly affect outcomes because it can compromise functional recovery ${ }^{[34]}$. Using a tourniquet can cause pain due to ischemiareperfusion injury ${ }^{[35]}$, particular when the tourniquet is used longer. The use of registration pins in robotassisted TKA increases pain even more.

We found that range of motion on PODs 1-3 was significantly restricted when a tourniquet was used, which is consistent with a report that tourniquets slow functional recovery after routine TKA ${ }^{[36]}$. Indeed, we found that using a tourniquet in robot-assisted TKA led to longer time to achieve the first straight-leg raise than what has been reported for patients after conventional TKA ${ }^{[37]}$, which likely reflects muscle damage as a result of prolonged tourniquet use. Indeed, the ischemia-reperfusion injury associated with tourniquets may significantly reduce the number of skeletal muscle fibers ${ }^{[38]}$. With a tourniquet may damage quadriceps, thereby delaying the recovery of muscle function, prolonging hospitalization and giving rise to long-lasting functional deficits ${ }^{[39]}$. However, we found no significant difference between the tourniquet and non-tourniquet groups in functional recovery up to 3 months after robot-assisted TKA. Studies with longer follow-up should verify and extend our results.

The use of a pneumatic tourniquet in conventional TKA may increase risk of postoperative complication $s^{[40,41]}$, although there is some evidence that it can protect against $\operatorname{DVT}^{[42,43]}$. We found that a tourniquet significantly increased the risk of tension blisters but not of other complications, even DVT. More work is needed to clarify the effects of tourniquet use on DVT and other complications after TKA, especially since the literature shows substantial heterogeneity of samples and study designs ${ }^{[3]}$. Clarifying these effects is particularly important for robot-assisted TKA because it involves longer operation and tourniquet time than routine TKA.

One of the strengths of our study is that it is a randomized, controlled, double-blinded trial. Another advantage is that all patients received the same standardized perioperative treatment, including 
multimodal analgesia, surgical procedure and blood loss management. This should reduce heterogeneity that could confound our analysis. On the other hand, our study was small and did not examine long-term clinical outcomes. The small sample may have failed to detect significant effects of a tourniquet on the incidence of complications, especially the DVT and pulmonary embolism. Large, multi-center studies are needed to evaluate the safety and efficacy of tourniquet use in robot-assisted TKA, especially now that robot-assisted surgery is becoming popular

\section{Conclusion}

Using a tourniquet in robot-assisted TKA does not appear to reduce total or hidden blood loss, but instead it appears to increase risk of more severe pain, longer length of stay, slower functional recovery, and complications. Therefore, we do not recommend tourniquets in robot-assisted TKA.

\section{Abbreviations}

TKA: Total knee arthroplasty

VAS: Visual analog scale

BMl: Body mass index

APTT: Activated partial thromboplastin time

PT: Prothrombin time

FIB: Fibrinogen

TT: Thrombin time

CT: Computed tomography

TXA: Tranexamic acid

HSS: Hospital for special surgery

PODs: Postoperative days

DVT: Deep vein thrombosis

Fig: Figure

\section{Declarations}


Yes. This study was approved by the ethics committee on clinical trial, west china hospital of Sichuan university and the institutional review board number was HX-IRB-AF-19-V4.0. This study was registered in the Chinese Clinical Trials Registry (ChiCTR2100041800). All patients provided consent for surgery and to have their data included in this study.

\section{Consent for publication}

All authors have stated for consents of publications. Availability of data and materials The data sets used and/or analyzed during the current study are fully available on reasonable request.

\section{Competing interest}

The authors have no relevant financial or non-financial interests to disclose. Funding This work was supported by 1.3.5 project for disciplines of excellence, West China Hospital, Sichuan University and Regional Innovation \& Cooperation program of Science \& Technology Department of Sichuan Province (No. 2021YFQ0028).

\section{Authors' contributions}

Yahao Lai and Hong Xu carried out the concepts, design, definition of intellectual content, data acquisition, data analysis and manuscript preparation. Xufeng Wan, Mingcheng Yuan and Qiang Su provided assistance for data acquisition, data analysis and statistical analysis. Zongke Zhou completed all operations and helped to performed manuscript review. All authors reviewed the final manuscript. All authors agree to be accountable for all aspects of the work. Acknowledgements

Not applicable

\section{Code availability}

Not applicable

\section{Publisher's Note}

Springer Nature remains neutral with regard to jurisdictional claims in published maps and institutional affiliations.

\section{References}


1. Abramoff B, Caldera FE. Osteoarthritis Pathology, Diagnosis, and Treatment Options. Medical Clinics of North America. 2020;104(2):293-+.

2. Sharma L. Osteoarthritis of the Knee. The New England journal of medicine. 2021;384(1):51-9.

3. Ritter MA, Faris PM, Keating EM, Meding JB. POSTOPERATIVE ALIGNMENT OF TOTAL KNEE REPLACEMENT - ITS EFFECT ON SURVIVAL. Clinical Orthopaedics and Related Research. 1994(299):153-6.

4. Grupp TM, Fritz B, Kutzner I, Schilling C, Bergmann G, Schwiesau J. Vitamin E stabilised polyethylene for total knee arthroplasty evaluated under highly demanding activities wear simulation. Acta Biomaterialia. 2017;48:415-22.

5. Riviere C, Iranpour F, Auvinet E, et al. Alignment options for total knee arthroplasty: A systematic review. Orthopaedics \& Traumatology-Surgery \& Research. 2017;103(7):1047-56.

6. Oussedik S, Abdel MP, Victor J, Pagnano MW, Haddad FS. Alignment in total knee arthroplasty WHAT'S IN A NAME? Bone \& Joint Journal. 2020;102B(3):276-9.

7. Lang JE, Mannava S, Floyd AJ, et al. Robotic systems in orthopaedic surgery. Journal of Bone and Joint Surgery-British Volume. 2011;93B(10):1296-9.

8. Liow MHL, Xia Z, Wong MK, Tay KJ, Yeo SJ, Chin PL. Robot-Assisted Total Knee Arthroplasty Accurately Restores the Joint Line and Mechanical Axis. A Prospective Randomised Study. Journal of Arthroplasty. 2014;29(12):2373-7.

9. Molli RG, Anderson KC, Buehler KC, Markel DC. Computer-Assisted Navigation Software Advancements Improve the Accuracy of Total Knee Arthroplasty. Journal of Arthroplasty. 2011;26(3):432-8.

10. Jacofsky DJ, Allen M. Robotics in Arthroplasty: A Comprehensive Review. Journal of Arthroplasty. 2016;31(10):2353-63.

11. Onggo JR, Onggo JD, De Steiger R, Hau R. Robotic-assisted total knee arthroplasty is comparable to conventional total knee arthroplasty: a meta-analysis and systematic review. Archives of Orthopaedic and Trauma Surgery. 2020;140(10):1533-49.

12. Ajwani SH, Jones M, Jarratt JW, Shepard GJ, Ryan WG. Computer assisted versus conventional total knee replacement: A comparison of tourniquet time, blood loss and length of stay. Knee. 2012;19(5):606-10.

13. Kalairajah Y, Cossey AJ, Verrall GM, Ludbrook G, Spriggins AJ. Are systemic emboli reduced in computer-assisted knee surgery? A prospective, randomised, clinical trial (vol 88-B, pg 198, 2006). Journal of Bone and Joint Surgery-British Volume. 2006;88B(10):1407-.

14. Kirkley A, Rampersaud R, Griffin S, Amendola A, Litchfield R, Fowler P. Tourniquet versus no tourniquet use in routine knee arthroscopy: A prospective, double-blind, randomized clinical trial. Arthroscopy. 2000;16(2):121-6.

15. Siebert W, Mai S, Kober R, Heeckt PE. Technique and first clinical results of robot-assisted total knee replacement. Knee. 2002;9(3):173-80. 
16. Vermue H, Luyckx T, de Grave PW, et al. Robot-assisted total knee arthroplasty is associated with a learning curve for surgical time but not for component alignment, limb alignment and gap balancing. Knee Surgery Sports Traumatology Arthroscopy. 2020.

17. Alcelik I, Pollock RD, Sukeik M, Bettany-Saltikov J, Armstrong PM, Eimer P. A Comparison of Outcomes With and Without a Tourniquet in Total Knee Arthroplasty A Systematic Review and Metaanalysis of Randomized Controlled Trials. Journal of Arthroplasty. 2012;27(3):331-40.

18. Huang Z, Xie X, Li L, et al. Intravenous and Topical Tranexamic Acid Alone Are Superior to Tourniquet Use for Primary Total Knee Arthroplasty A Prospective, Randomized Controlled Trial. Journal of Bone and Joint Surgery-American Volume. 2017;99(24):2053-61.

19. Xu H, Yang J, Xie J, et al. Tourniquet use in routine primary total knee arthroplasty is associated with a higher transfusion rate and longer postoperative length of stay: a real-world study. Bmc Musculoskeletal Disorders. 2020;21(1).

20. Bellemans J, Vandenneucker $\mathrm{H}$, Vanlauwe J. Robot-assisted total knee arthroplasty. Clinical Orthopaedics and Related Research. 2007(464):111-6.

21. Cai DF, Fan $Q H$, Zhong $H H$, Peng S, Song $H$. The effects of tourniquet use on blood loss in primary total knee arthroplasty for patients with osteoarthritis: a meta-analysis. Journal of Orthopaedic Surgery and Research. 2019;14(1).

22. Nadler SB, Hidalgo JU, Bloch T. PREDICTION OF BLOOD VOLUME IN NORMAL HUMAN ADULTS. Surgery. 1962;51(2):224-32.

23. Gross JB. ESTIMATING ALLOWABLE BLOOD-LOSS - CORRECTED FOR DILUTION. Anesthesiology. 1983;58(3):277-80.

24. Liu D, Graham D, Gillies K, Gillies RM. Effects of tourniquet use on quadriceps function and pain in total knee arthroplasty. Knee surgery \& related research. 2014;26(4):207-13.

25. Goel R, Rondon AJ, Sydnor K, et al. Tourniquet Use Does Not Affect Functional Outcomes or Pain After Total Knee Arthroplasty. Journal of Bone and Joint Surgery-American Volume. 2019;101(20):1821-8.

26. Liu X, Zhang X, Chen Y, Wang Q, Jiang Y, Zeng B. Hidden Blood Loss After Total Hip Arthroplasty. Journal of Arthroplasty. 2011;26(7):1100-5.

27. Pua Y-H. The Time Course of Knee Swelling Post Total Knee Arthroplasty and Its Associations with Quadriceps Strength and Gait Speed. Journal of Arthroplasty. 2015;30(7):1215-9.

28. Huang ZY, Pei FX, Ma J, et al. Comparison of three different tourniquet application strategies for minimally invasive total knee arthroplasty: a prospective non-randomized clinical trial. Archives of Orthopaedic and Trauma Surgery. 2014;134(4):561-70.

29. Lee OS, Lee MC, Han H-S. Efficacy and safety of a new elastic tourniquet cuff in total knee arthroplasty: a prospective randomized controlled study. Biomedical Engineering Online. 2017;16.

30. Patel NK, Johns W, Vedi V, Langstaff RJ, Golladay GJ. Tourniquet and tranexamic acid use in total knee arthroplasty. Arthroplasty today. 2020;6(2):246-50. 
31. Jeon S-W, Kim K-I, Song SJ. Robot-Assisted Total Knee Arthroplasty Does Not Improve Long-Term Clinical and Radiologic Outcomes. Journal of Arthroplasty. 2019;34(8):1656-61.

32. Horlocker TT, Hebl JR, Gali B, et al. Anesthetic, patient, and surgical risk factors for neurologic complications after prolonged total tourniquet time during total knee arthroplasty. Anesthesia and Analgesia. 2006;102(3):950-5.

33. Arthur JR, Spangehl MJ. Tourniquet Use in Total Knee Arthroplasty. Journal of Knee Surgery. 2019;32(8):719-29.

34. Oktas B, Vergili O. The effect of intensive exercise program and kinesiotaping following total knee arthroplasty on functional recovery of patients. Journal of Orthopaedic Surgery and Research. 2018;13.

35. Huang Z, Ma J, Zhu Y, et al. Timing of Tourniquet Release in Total Knee Arthroplasty. Orthopedics. 2015;38(7):445-51.

36. Liu Y, Si H, Zeng Y, Li M, Xie H, Shen B. More pain and slower functional recovery when a tourniquet is used during total knee arthroplasty. Knee Surgery Sports Traumatology Arthroscopy. 2020;28(6):1842-60.

37. Zhou K, Ling T, Wang H, et al. Influence of tourniquet use in primary total knee arthroplasty with drainage: a prospective randomised controlled trial. Journal of Orthopaedic Surgery and Research. $2017 ; 12$.

38. Bushell AJ, Klenerman L, Taylor S, et al. Ischaemic preconditioning of skeletal muscle 1. Protection against the structural changes induced by ischaemia/reperfusion injury. Journal of Bone and Joint Surgery-British Volume. 2002;84B(8):1184-8.

39. Meier W, Mizner R, Marcus R, Dibble L, Peters C, Lastayo PC. Total knee arthroplasty: Muscle impairments, functional limitations, and recommended rehabilitation approaches. Journal of Orthopaedic \& Sports Physical Therapy. 2008;38(5):246-56.

40. Abdelsalam A, Eyres KS. EFFECTS OF TOURNIQUET DURING TOTAL KNEE ARTHROPLASTY - A PROSPECTIVE RANDOMIZED STUDY. Journal of Bone and Joint Surgery-British Volume. $1995 ; 77 B(2): 250-3$.

41. Clarke MT, Longstaff L, Edwards D, Rushton N. Tourniquet-induced wound hypoxia after total knee replacement. Journal of Bone and Joint Surgery-British Volume. 2001;83B(1):40-4.

42. Aglietti P, Baldini A, Vena LM, Abbate R, Fedi S, Falciani M. Effect of tourniquet use on activation of coagulation in total knee replacement. Clinical Orthopaedics and Related Research. 2000(371):16977.

43. Fahmy NR, Patel DG. HEMOSTATIC CHANGES AND POSTOPERATIVE DEEP-VEIN THROMBOSIS ASSOCIATED WITH USE OF A PNEUMATIC TOURNIQUET. Journal of Bone and Joint SurgeryAmerican Volume. 1981;63(3):461-5.

\section{Tables}


Due to technical limitations, tables are only available as a download in the Supplemental Files section.

\section{Figures}

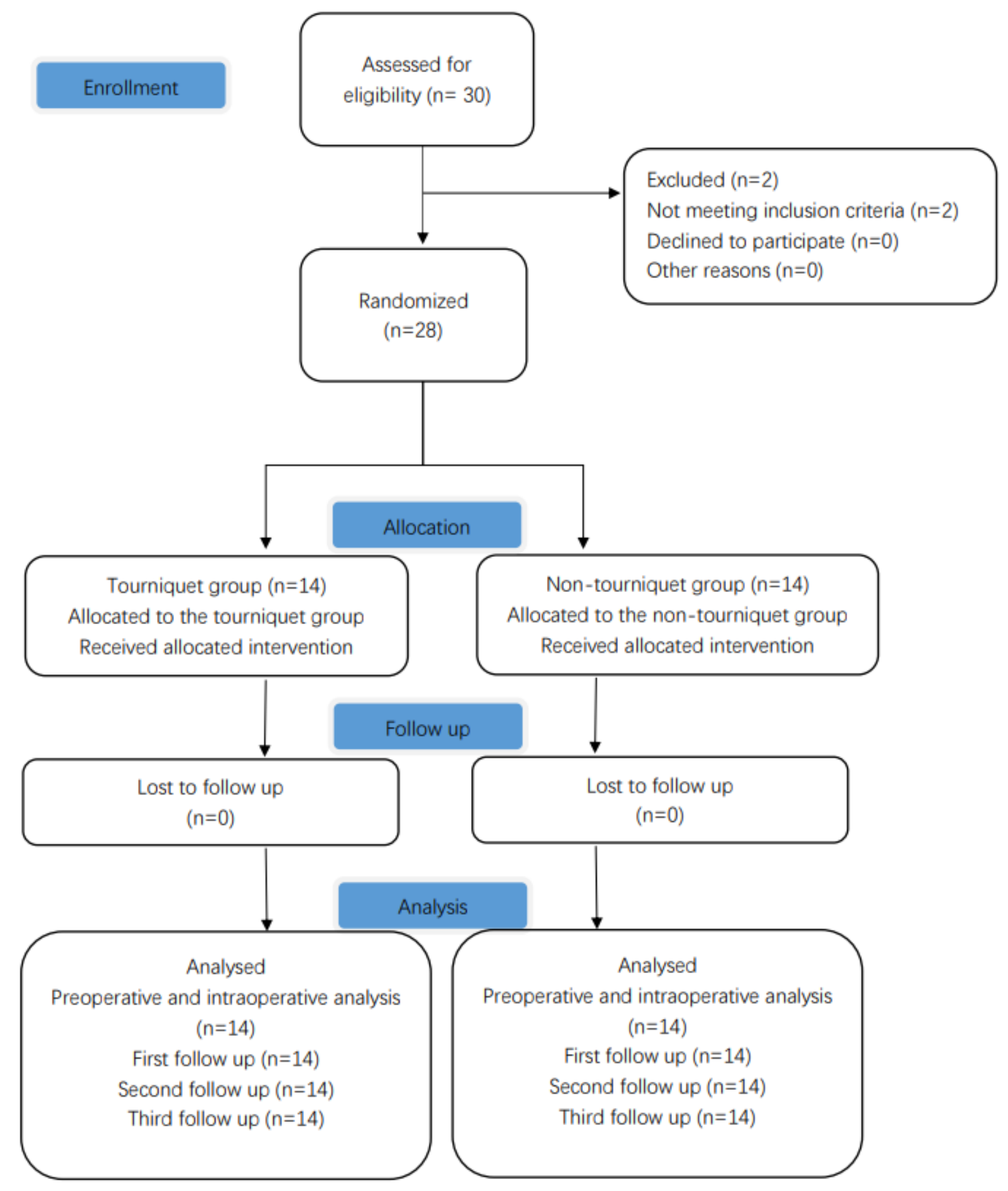

Figure 1

Flow diagram of patient enrollment and analysis. 

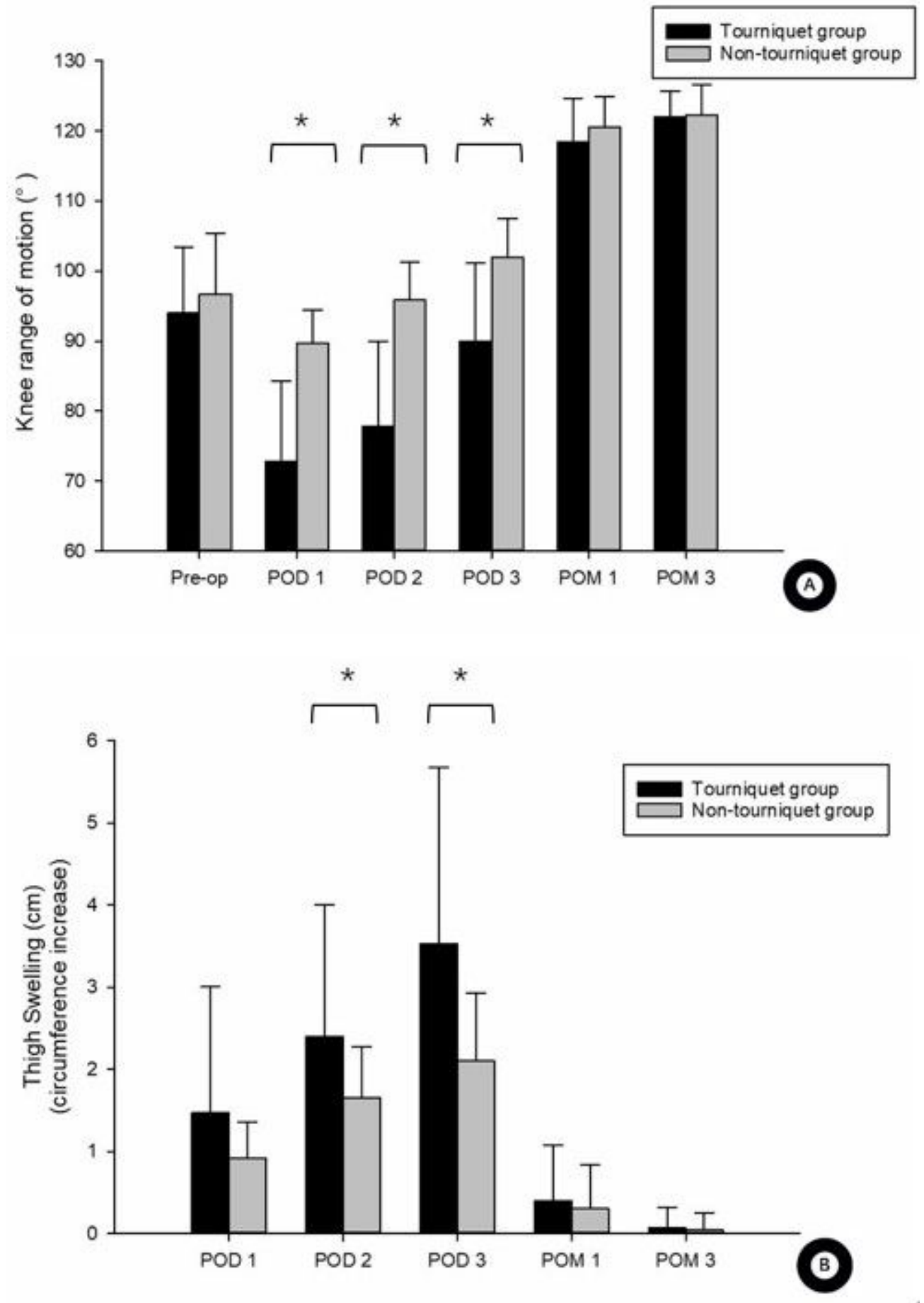

Figure 2

Differences in $(A)$ knee range of motion and $(B)$ thigh swelling between patients who underwent robotassisted TKA with or without a tourniquet. ${ }^{*}<0.05$. Abbreviations: $P O D$, postoperative day; POM, postoperative month; Pre-op, preoperative. 

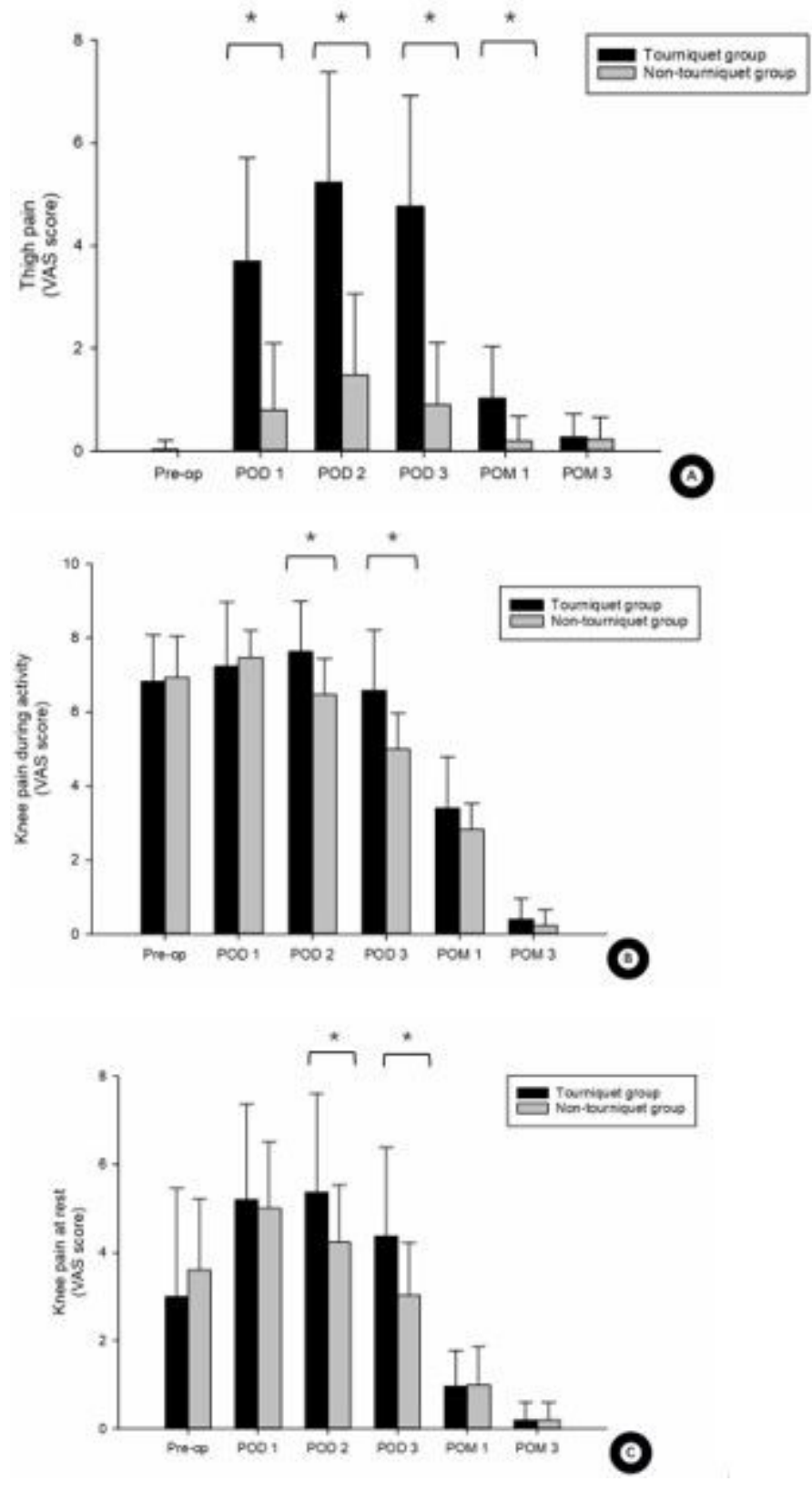

Figure 3

Differences in (A) thigh pain, (B) knee pain during activity and (C) knee pain at rest. ${ }^{*} \mathrm{P}<0.05$. Abbreviations: VAS, visual analog scale. Pre-op, preoperative; POD, postoperative day; POM, postoperative month;

\section{Supplementary Files}

This is a list of supplementary files associated with this preprint. Click to download.

- Table1.docx

- Table2.docx

- Table3.docx 